\title{
Article \\ Symmetries and Solvability of a Class of Higher Order Systems of Ordinary Difference Equations
}

\author{
Kgatliso Mkhwanazi ${ }^{\dagger}$ and Mensah Folly-Gbetoula *, $+\mathbb{D}$ \\ School of Mathematics, University of the Witwatersrand, Johannesburg 2050, South Africa; \\ 1477029@students.wits.ac.za \\ * Correspondence: Mensah.Folly-Gbetoula@wits.ac.za \\ + These authors contributed equally to this work.
}

\begin{abstract}
We perform Lie analysis for a system of higher order difference equations with variable coefficients and derive non-trivial symmetries. We use these symmetries to find exact formulas for the solutions in terms of $k$. Furthermore, a detailed study for a specific value of $k$ is presented. Our findings generalize some results in the literature.
\end{abstract}

Keywords: difference equations; symmetry; exact solutions

\section{Introduction}

A Norwegian mathematician, Marius Sophus Lie (1842-1899) is responsible for the discovery of the transformations of variables and its properties. He began by investigating the continuous groups of transformations that would leave the differential equations invariant and his work created what is now known as symmetry analysis of differential equations. Lie's original aim was to set a general theory for the integration of ordinary differential equations which was similar to the work that was done by Galois Abel [1] on algebraic equations in 1888. During the nineteenth century, Lie developed and applied the symmetry analysis of differential equations [2]. The theory that he developed enabled one to derive the solutions of differential equations in an algorithmic way that did not require any special guesses. There has been a lot of interest in the way Lie approached differential equations and notable mentions include the work done by Sedov Leonid Ivanovich [3] and Garrett Birkhoff [4] on the dimensional analysis. Their individual work proved to be important in the understanding of Lie's approach to differential equations because they showed that Lie's theory gave pertinent results in applied problems. Before this, there was a German mathematician Hermann Weyl (1885-1955) who in 1928 took interest in the abstract theory of Lie groups, and the term Lie group was coined by him in the same year. There has been a restoration of interest in Lie's theory in recent decades and during these decades there has been a lot of crucial progress that was made from an applied point of view or a theoretical one. Lie's theory involves a lot of tedious and cumbersome calculations. Lie group analysis has been and is still an essential tool in various fields like physics, number theory, differential equations, differential geometry, analysis and more.

Many researchers have investigated the application of Lie symmetry analysis to difference equations. Systematic algorithms and methods dealing with the derivation of symmetries for difference equations are now recorded and well documented. Some of the first works can be traced back to Maeeda [5,6] who developed an algorithm for obtaining continuous point symmetries of ordinary difference equations. Levi and Winternitz [7], Hydon [8], Quispel and Sahadeva [9], among others, have greatly contributed to the use of the Lie's theory to difference equations.

Just like differential equations, difference equations have applications in real life (simple and compound interests, loan repayments, biological population dynamics, etc.). In general, difference equations are the perfect tools for describing phenomena that happen in discrete time steps. For example, in biological populations without overlapping 
generations, the growth of population takes place in discrete time steps and is modeled by difference equations (see [10]). An example of a typical model is that of semelparous populations which are insect populations with a single reproductive period before death. If we denote by $N_{n}$ the number of adults in the $n$th breeding time and $a_{0}$ the average number of eggs laid by an adult, the model turns out to be

$$
N_{n+1}=\frac{a_{0} N_{n}}{1+t N_{n}}
$$

for some constant $t$. See [10] for more details. Let $\kappa$ denote the carrying capacity of the environment. Then, $t=\frac{a_{0}-1}{\kappa}$, and the model is known as the Beverton-Holt model [10]. Note that (1) implies that

$$
N_{n+2}=\frac{N_{n}}{a+b N_{n}},
$$

where $a=1 / a_{0}^{2}$ and $b=t\left(a_{0}+1\right) / a_{0}^{2}$.

In [11], the authors solved and dealt with the recursive equations:

$$
x_{n+1}=\frac{x_{n-1} y_{n-3}}{y_{n-1}\left(1+x_{n-1} y_{n-3}\right)}, \quad y_{n+1}=\frac{y_{n-1} x_{n-3}}{x_{n-1}\left( \pm 1 \pm y_{n-1} x_{n-3}\right)},
$$

where the real numbers $x_{-3}, x_{-2}, x_{-1}, x_{0}, y_{-3}, y_{-2}, y_{-1}$ and $y_{0}$ are the initial conditions.

In [12], the author determined and formulated the analytical solutions of the rational recursive equations:

$$
x_{n+1}=\frac{x_{n-1} y_{n-3}}{y_{n-1}\left( \pm 1 \mp x_{n-1} y_{n-3}\right)}, \quad y_{n+1}=\frac{y_{n-1} x_{n-3}}{x_{n-1}\left(\mp 1 \pm y_{n-1} x_{n-3}\right)},
$$

where $x_{-3}, x_{-2}, x_{-1}, x_{0}, y_{-3}, y_{-2}, y_{-1}$ and $y_{0}$ are the initial conditions, which are arbitrary non-zero real numbers.

Note that appropriate change of variables transform equations in (3) and (4) into equations similar to (2).

The aim of this study is to generalize the results in [11,12] by studying the system of ordinary difference equations

$$
u_{n+1}=\frac{u_{n-1} v_{n-k-1}}{v_{n-k+1}\left(A_{n}+B_{n} u_{n-1} v_{n-k-1}\right)}, v_{n+1}=\frac{u_{n-k-1} v_{n-1}}{u_{n-k+1}\left(C_{n}+D_{n} u_{n-k-1} v_{n-1}\right)},
$$

where $A_{n}, B_{n}, C_{n}$ and $D_{n}$ are real sequences, using a symmetry method. Note that $u_{-k-1}, \ldots, u_{0}, v_{-k-1}, \ldots, v_{0}$ are the initial conditions. For a similar work, see [13].

Understanding Lie groups and being able to use them is important because there is a lot that can be done with Lie groups, for example, we can get the Lie algebra action for a linear object by getting the derivative of the Lie group action. This is useful because when it comes to linear objects, it is much simpler to work with a Lie algebra than directly working with a Lie group. This is just one example of the many useful ways Lie groups can be used.

\section{Preliminaries}

In this section, we introduce some basic definitions and theorems needed in the work. Most of our definitions can be found in $[8,14]$.

Definition 1. A forward $O \Delta E$ has the form

$$
u_{n+k}=\omega\left(n, u_{n}, u_{n+1}, \ldots, u_{n+k-1}\right), \quad n \in D,
$$

The domain $D$ of the forward $O \Delta E$ is said to be a regular domain if $\omega_{, u_{n}} \neq 0, n \in D$. Here, the notation $\omega, u_{n}$ stands for $\frac{\partial \omega}{\partial u_{n}}$. 
Definition 2. A parametrized set of point transformations,

$$
\Gamma_{\varepsilon}: x \mapsto \hat{x}(x ; \varepsilon), \quad \varepsilon \in\left(\varepsilon_{0}, \varepsilon_{1}\right),
$$

where $\varepsilon_{0}<0<\varepsilon_{1}$, is a one-parameter local Lie group if the following conditions are satisfied:

1. $\Gamma_{0}$ is the identity map, so that $\hat{x}=x$ when $\varepsilon=0$.

2. $\quad \Gamma_{\delta} \Gamma_{\varepsilon}=\Gamma_{\delta+\varepsilon}$ for every $\delta, \varepsilon$ sufficiently close to zero.

3. Each $\hat{x}_{\alpha}$ can be represented as Taylor series in $\varepsilon$ (in a neighborhood of $\varepsilon=0$ that is determined by $x$ ), and therefore

$$
\hat{x}_{\alpha}(x ; \varepsilon)=x_{\alpha}+\varepsilon \xi_{\alpha}(x)+O\left(\varepsilon^{2}\right), \alpha=1, \ldots, N .
$$

Definition 3. Consider $\tilde{u}=U(u, \varepsilon)$, with $\varepsilon=0$, expand in Taylor series,

$$
\tilde{u}=u+\varepsilon\left(\left.\frac{\partial U}{\partial \varepsilon}\right|_{\varepsilon=0}\right)+\frac{\varepsilon^{2}}{2 !}\left(\left.\frac{\partial^{2} U}{\partial \varepsilon^{2}}\right|_{\varepsilon=0}\right)+\ldots
$$

up to first order, we have

$$
\tilde{u}=u+\varepsilon\left(\left.\frac{\partial U}{\partial \varepsilon}\right|_{\varepsilon=0}\right)+O\left(\varepsilon^{2}\right) .
$$

Definition 4. A symmetry generator of (6) is denoted by $U$ and is given by

$$
U=Q \frac{\partial}{\partial u_{n}}+S Q \frac{\partial}{\partial u_{n+1}}+\cdots+S^{k-1} Q \frac{\partial}{\partial u_{n+k-1}},
$$

where $Q$ is the characteristic of the group of transformations.

In the above definition, $S^{j} Q\left(n, u_{n}\right)=Q\left(n+j, u_{n+j}\right)$. The operator $S$ is known as the shift operator.

Consider the system of ordinary difference equations of the form

$$
u_{n+k+2}=\omega_{1}\left(u_{n+k}, v_{n}, v_{n+2}\right), v_{n+k+2}=\omega_{2}\left(u_{n}, u_{n+2}, v_{n+k}\right) \text {, }
$$

where the independent variable is $n$ and the dependent variables are $u_{n}, v_{n}$ and their shifts.

Consider the group of transformations

$$
\begin{aligned}
& \left(n, u_{n}, v_{n}\right) \mapsto\left(n, \tilde{u}_{n}=u_{n}+\varepsilon Q_{1}\left(n, u_{n}\right)\right. \\
& \left.\quad+O\left(\varepsilon^{2}\right), \tilde{v}_{n}=v_{n}+\varepsilon Q_{2}\left(n, v_{n}\right)+O\left(\varepsilon^{2}\right)\right) .
\end{aligned}
$$

In (13), the characteristic of the group of transformations is $Q=\left(Q_{1}, Q_{2}\right)$. The infinitesimal generator corresponds to

$$
U=Q_{1} \partial_{u_{n}}+Q_{2} \partial_{v_{n}}
$$

where $\partial_{x}=\frac{\partial}{\partial x}$. In this work, we will need the $k$ th extension

$$
U^{[k]}=Q_{1} \partial_{u_{n}}+Q_{2} \partial_{v_{n}}+S^{2} Q_{1} \partial_{u_{n+2}}+S^{2} Q_{2} \partial_{v_{n+2}}+S^{k} Q_{1} \partial_{u_{n+k}}+S^{k} Q_{2} \partial_{v_{n+k}}
$$

of (14).

For the set of solutions of (12) to be mapped to itself, the following linearized symmetry conditions

$$
S^{(k+2)} Q_{1}-U^{[k]} \omega_{1}=0 \text { and } S^{(k+2)} Q_{2}-U^{[k]} \omega_{2}=0,
$$


whenever (12) is true, must be satisfied. If the conditions given in (16), that is, $Q_{j}\left(n+k+2, \Omega_{j}\right)-U^{[k]}\left(\Omega_{j}\right)=0, j=1,2$, are satisfied, then the group of transformations (13) is a symmetry group.

\section{Symmetries and Solutions of the System of Difference Equations}

Equivalently, Equation (5) can be written as

$$
\begin{aligned}
& u_{n+k+2}=\omega_{1}=\frac{u_{n+k} v_{n}}{v_{n+2}\left(a_{n}+b_{n} u_{n+k} v_{n}\right)}, \\
& v_{n+k+2}=\omega_{2}=\frac{u_{n} v_{n+k}}{u_{n+2}\left(c_{n}+d_{n} u_{n} v_{n+k}\right)},
\end{aligned}
$$

where $a_{n}, b_{n}, c_{n}$ and $d_{n}$ are real sequences. Applying (16) to (17) yields

$$
\begin{aligned}
& -S^{k+2} Q_{1}+\frac{a_{n} u_{n+k} Q_{2}}{v_{n+2}\left(a_{n}+b_{n} u_{n+k} v_{n}\right)^{2}}- \\
& \frac{u_{n+k} v_{n} S^{2} Q_{2}}{v_{n+2}^{2}\left(a_{n}+b_{n} u_{n+k} v_{n}\right)}+\frac{a_{n} v_{n} S^{k} Q_{1}}{v_{n+2}\left(a_{n}+b_{n} u_{n+k} v_{n}\right)^{2}}=0, \\
& -S^{k+2} Q_{2}+\frac{c_{n} v_{n+k} Q_{1}}{u_{n+2}\left(c_{n}+d_{n} u_{n} v_{n+k}\right)^{2}}- \\
& \frac{u_{n} v_{n+k} S^{2} Q_{1}}{u_{n+2}^{2}\left(c_{n}+d_{n} u_{n} v_{n+k}\right)}+\frac{c_{n} u_{n} S^{k} Q_{2}}{u_{n+2}\left(c_{n}+d_{n} u_{n} v_{n+k}\right)^{2}}=0 .
\end{aligned}
$$

After a set of long calculations, we get a system of determining equations for the characteristics $Q_{1}$ and $Q_{2}$. Solving this system, we get

$$
Q_{2}\left(n, v_{n}\right)=\lambda_{n} v_{n} \quad \text { and } \quad Q_{1}\left(n, u_{n}\right)=\alpha_{n} u_{n}
$$

provided that

$$
\alpha_{n}+\lambda_{n+k}=0 \text { and } \lambda_{n}+\alpha_{n+k}=0
$$

Using (21), we have that

$$
\alpha_{n+2 k}-\alpha_{n}=0 \text {. }
$$

The characteristic equation corresponding to (22) is giving by

$$
r^{2 k}-1=0 \text {. }
$$

Basically, $r$ are the $2 k$-th roots of 1 , which are obtained as follows:

$$
r^{2 k}=e^{i(0+2 p \pi)},
$$

that is,

$$
r=e^{i\left(\frac{2 p \pi}{2 k}\right)}
$$

for any $2 k$ successive values of $p \in \mathbb{Z}$, say $0 \leq p \leq 2 k-1$. This is the same as saying that

$$
r=e^{i\left(\frac{p \pi}{k}\right)}, \quad 0 \leq p \leq 2 k-1 \text {. }
$$


It follows that the solutions $\alpha_{n}$ of (22) are given by

$$
\alpha_{n}=r^{n}=e^{i\left(\frac{n p \pi}{k}\right)}, \quad 0 \leq p \leq 2 k-1
$$

Using (21), we have that

$$
\begin{aligned}
\lambda_{n} & =-\alpha_{n+k} \\
& =-e^{\frac{i(n+k) p \pi}{k}}, \quad 0 \leq p \leq 2 k-1 .
\end{aligned}
$$

Therefore (thanks to (14), (20), (27) and (28)), the system (17) has the following $2 k$ symmetry generators:

$$
U=e^{i p \pi n / k} u_{n} \frac{\partial}{\partial u_{n}}-e^{i p \pi(n+k) / k_{v_{n}}} \frac{\partial}{\partial v_{n}}
$$

where $0 \leq p \leq 2 k-1$. Thanks to (20), the canonical coordinates [15] are given by

$$
s_{n}^{1}=\int \frac{d u_{n}}{Q_{1}\left(n, u_{n}\right)}=\frac{1}{\alpha_{n}} \ln \left|u_{n}\right| \quad \text { and } \quad s_{n}^{2}=\int \frac{d v_{n}}{Q_{2}\left(n, v_{n}\right)}=\frac{1}{\lambda_{n}} \ln \left|v_{n}\right| .
$$

Motivated by (21), we let $\widetilde{Y}_{n}=\alpha_{n} s_{n}^{1}+\lambda_{n+k} s_{n+k}^{2}=\ln \left|u_{n} v_{n+k}\right|$ and $\widetilde{X}_{n}=\lambda_{n} s_{n}^{2}+$ $\alpha_{n+k} s_{n+k}^{1}=\ln \left|v_{n} u_{n+k}\right|$. For a better understanding, we use $X_{n}=\exp \left\{-\widetilde{X}_{n}\right\}$ and $Y_{n}=\exp \left\{-\widetilde{Y}_{n}\right\}$. We then get the invariants

$$
X_{n}=\frac{1}{v_{n} u_{n+k}} \quad \text { and } \quad Y_{n}=\frac{1}{u_{n} v_{n+k}}
$$

Here, (31) is invariant under the group transformations of (20). In other words, the action of the symmetry generators, given in (29), on (31) gives zero. It is worthwhile mentioning that the symmetries together with the constraints on $\alpha_{n}$ and $\lambda_{n}$ have helped us to come up with the appropriate change of variables that will lead to the reduced system of equations. This is just one of the many roles of symmetries.

Using (31), (5) is reduced to a second-order difference equations:

$$
X_{n+2}=a_{n} X_{n}+b_{n} \text { and } Y_{n+2}=c_{n} Y_{n}+d_{n}
$$

The closed form solutions of (32) are, respectively, as follows:

$$
\begin{aligned}
& X_{2 n+i}=X_{i}\left(\prod_{k_{1}=0}^{n-1} a_{2 k_{1}+i}\right)+\sum_{l=0}^{n-1}\left(b_{2 l+i} \prod_{k_{2}=l+1}^{n-1} a_{2 k_{2}+i}\right), \\
& Y_{2 n+i}=Y_{i}\left(\prod_{k_{1}=0}^{n-1} c_{2 k_{1}+i}\right)+\sum_{l=0}^{n-1}\left(d_{2 l+i} \prod_{k_{2}=l+1}^{n-1} c_{2 k_{2}+i}\right),
\end{aligned}
$$

for $i=0,1$.

From (31), we get that

$$
u_{n+2 k}=\frac{Y_{n}}{X_{n+k}} u_{n} \text { and } v_{n+2 k}=\frac{X_{n}}{Y_{n+k}} v_{n}
$$

Performing iterations on (35), respectively, leads to the following:

$$
u_{2 k n+j}=u_{j} \prod_{m=0}^{n-1} \frac{Y_{2 k m+j}}{X_{2 k m+k+j}} \text { and } v_{2 k n+j}=v_{j} \prod_{m=0}^{n-1} \frac{X_{2 k m+j}}{Y_{2 k m+k+j}},
$$


where $j=0,1,2, \ldots, 2 k-1$.

The subscript of $X$ and $Y$ in (33) and (34) is $2 n+1$, where $i=0,1$ but the ones of $X$ and $Y$ in (36) are $2 k m+j$ and $2 k m+k+j$, where $j=0,1,2, \ldots, 2 k-1$. Therefore, we want to write $2 k m+j$ and $2 k m+k+j$ in the form similar to $2 n+i$. We know that any positive integer $r$ can be written as $r=k\lfloor r / k\rfloor+\tau(r), 0 \leq \tau(r) \leq k-1$, where $\tau(r)$ denotes the remainder when $r$ is divided by $k$. Hence,

$$
2 k m+j=2\left(k m+\left\lfloor\frac{j}{2}\right\rfloor\right)+\tau(j)
$$

and

$$
2 k m+k+j=2\left(k m+\left\lfloor\frac{k+j}{2}\right\rfloor\right)+\tau(k+j) .
$$

Now, (37) and (38) are of the form similar to $2 n+i$, as $\tau(j)$ and $\tau(k+j)$ will either be 0 or 1 . Substituting (37) and (38) into (36) leads to the following:

$$
u_{2 k n+j}=u_{j} \prod_{m=0}^{n-1} \frac{Y_{2\left(k m+\left\lfloor\frac{j}{2}\right\rfloor\right)+\tau(j)}}{X_{2\left(k m+\left\lfloor\frac{k+j}{2}\right\rfloor\right)+\tau(k+j)}}
$$

and

$$
v_{2 k n+j}=v_{j} \prod_{m=0}^{n-1} \frac{X_{2\left(k m+\left\lfloor\frac{j}{2}\right\rfloor\right)+\tau(j)}}{Y_{2\left(k m+\left\lfloor\frac{k+j}{2}\right\rfloor\right)+\tau(k+j)}} .
$$

Using (33), (34) (39) and (40), we get the following:

$$
\begin{aligned}
& u_{2 k n+j}=u_{j} \prod_{m=0}^{n-1}
\end{aligned}
$$

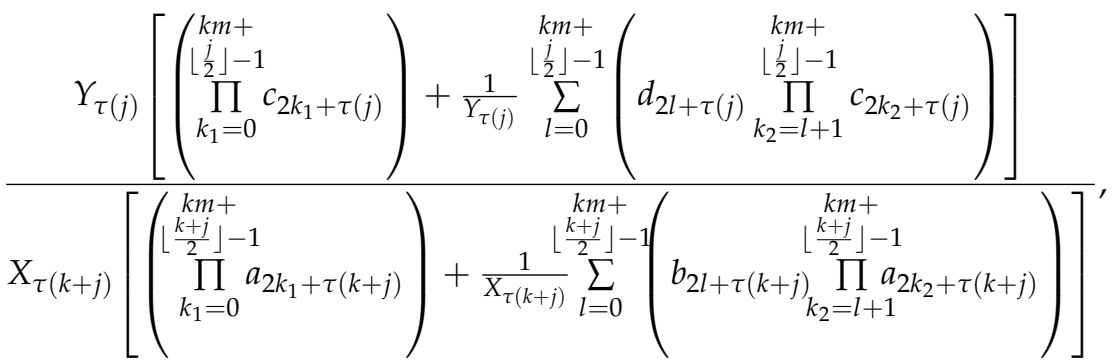

that is,

$$
\begin{aligned}
& u_{2 k n+j}=u_{j} \prod_{m=0}^{n-1} \frac{u_{\tau(k+j)+k} v_{\tau(k+j)}}{u_{\tau(j)} v_{\tau(j)+k}} \\
& \frac{\left(\prod_{k_{1}=0}^{\substack{k m+\\
\left\lfloor\frac{j}{2}\right\rfloor-1}} c_{2 k_{1}+\tau(j)}\right)+u_{\tau(j)} v_{\tau(j)+k} \sum_{l=0}^{\substack{k m+\\
\left\lfloor\frac{j}{2}\right\rfloor-1}}\left(d_{2 l+\tau(j)} \prod_{k_{2}=l+1}^{\substack{k m+\\
\left\lfloor\frac{j}{2}\right\rfloor-1}} c_{2 k_{2}+\tau(j)}\right)}{\left(\prod_{k_{1}=0}^{\left\lfloor\frac{k m+}{2}\right\rfloor-1} a_{2 k_{1}+\tau(k+j)}\right)+u_{\tau(k+j)+k} v_{\tau(k+j)} \sum_{l=0}^{\left\lfloor\frac{k m+j}{2}\right\rfloor-1}\left(\begin{array}{c}
k m+ \\
\left\lfloor\frac{k+j}{2}\right\rfloor-1 \\
\left.b_{2 l+\tau(k+j)} \prod_{k_{2}=l+1} a_{2 k_{2}+\tau(k+j)}\right)
\end{array}\right)}
\end{aligned}
$$


and, similarly,

$$
\begin{aligned}
& v_{2 k n+j}=v_{j} \prod_{m=0}^{n-1} \frac{u_{\tau(k+j)} v_{\tau(k+j)+k}}{u_{\tau(j)+k} v_{\tau(j)}}
\end{aligned}
$$

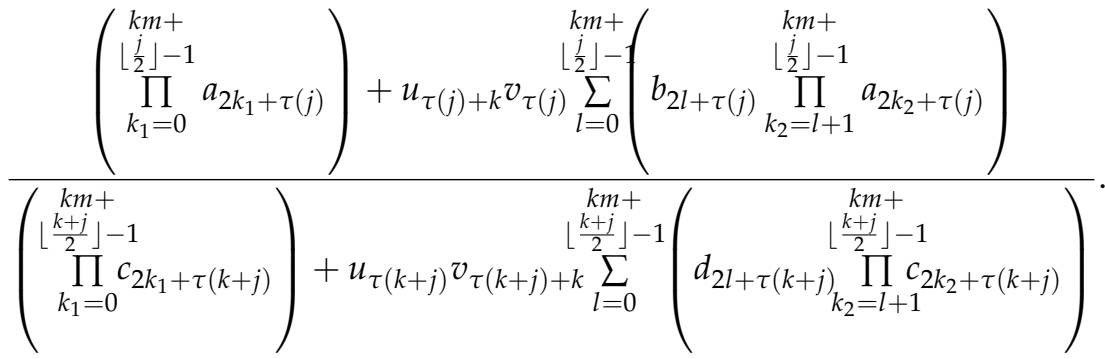

Back-shifting the equations above by $k$ yields

$$
\begin{aligned}
& u_{2 k n+j-k}=u_{j-k} \prod_{m=0}^{n-1} \frac{u_{\tau(k+j)} v_{\tau(k+j)-k}}{u_{\tau(j)-k} v_{\tau(j)}}
\end{aligned}
$$

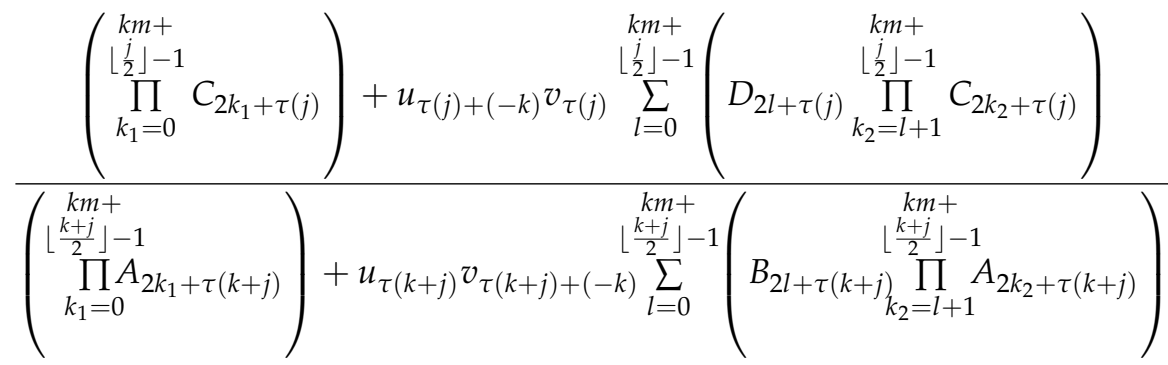

and

$$
\begin{aligned}
& v_{2 k n+j-k}=v_{j-k} \prod_{m=0}^{n-1} \frac{u_{\tau(k+j)-k} v_{\tau(k+j)}}{u_{\tau(j)} v_{\tau(j)-k}} \\
& \left(\prod_{k_{1}=0}^{\substack{k m+\\
\left\lfloor\frac{j}{2}\right\rfloor-1}} A_{2 k_{1}+\tau(j)}\right)+u_{\tau(j)} v_{\tau(j)+(-k)} \sum_{l=0}^{\substack{\left.k m+\\
\frac{j}{2}\right\rfloor-1}}\left(\begin{array}{c}
\substack{k m+\\
\left\lfloor\frac{j}{2}\right\rfloor-1} \\
\left.B_{2 l+\tau(j)} \prod_{k_{2}=l+1} A_{2 k_{2}+\tau(j)}\right)
\end{array}\right)
\end{aligned}
$$

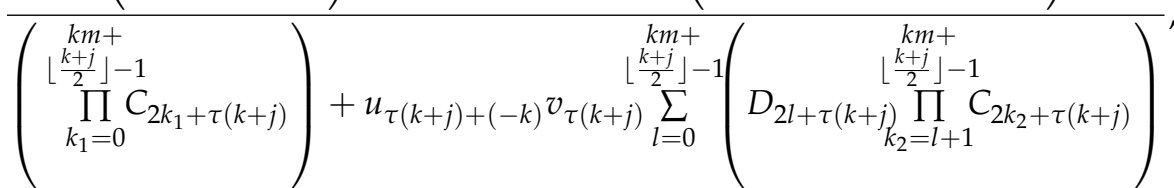

for $j=0,1,2, \ldots, 2 k-1$. Equations (43) and (44) are the solutions of (5).

\subsection{The Case When $A_{n}, B_{n}, C_{n}$ and $D_{n}$ Are Constant Sequences}

Letting $A_{n}=A, B_{n}=B, C_{n}=C$ and $D_{n}=D$ for all $n$ simplifies (43) and (44) as follows:

$$
u_{j-k} \prod_{m=0}^{n-1} \frac{u_{\tau(k+j)} v_{\tau(k+j)-k}}{u_{\tau(j)-k} v_{\tau(j)}} \frac{C^{k m+\left\lfloor\frac{j}{2}\right\rfloor}+D u_{\tau(j)+(-k)} v_{\tau(j)} \sum_{\substack{l=0 \\ l j}}^{\substack{k m+\\ 2}} C^{l}}{A^{k m+\left\lfloor\frac{k+j}{2}\right\rfloor}+B u_{\tau(k+j)} v_{\tau(k+j)+(-k)} \sum_{l=0}^{\left\lfloor\frac{k+j}{2}\right\rfloor-1} A^{l}}
$$


and

$$
v_{j-k} \prod_{m=0}^{n-1} \frac{u_{\tau(k+j)-k} v_{\tau(k+j)}}{u_{\tau(j)} v_{\tau(j)-k}} \frac{A^{k m+\left\lfloor\frac{j}{2}\right\rfloor}+B u_{\tau(j)} v_{\tau(j)+(-k)} \sum_{l=0}^{k m+\left\lfloor\frac{j}{2}\right\rfloor-1} A^{l}}{C^{k m+\left\lfloor\frac{k+j}{2}\right\rfloor}+D u_{\tau(k+j)+(-k)} v_{\tau(k+j)} \sum_{l=0}^{\left\lfloor\frac{k+j}{2}\right\rfloor-1} C^{l}},
$$

where $j=0,1,2, \ldots, 2 k-1$.

\subsection{A Detailed Study of the Case $k=2$}

One of the aims of this section is to verify the results in [11,12]. To achieve this, we substitute $k=2$ into (45) and (46). It yields the following:

$$
u_{4 n+j-2}=u_{j-2} \prod_{m=0}^{n-1} \frac{u_{\tau(2+j)} v_{\tau(2+j)-2}}{u_{\tau(j)-2} v_{\tau(j)}} \frac{C^{2 m+\left\lfloor\frac{j}{2}\right\rfloor}+D u_{\tau(j)+(-2)} v_{\tau(j)} \sum_{l=0}^{\substack{\left.i \frac{j}{2}\right\rfloor-1 \\ C^{l}}}}{A^{2 m+\left\lfloor\frac{2+j}{2}\right\rfloor}+B u_{\tau(2+j)} v_{\tau(2+j)-2} \sum_{l=0}^{\lfloor+j} A^{l}},
$$

and

$$
v_{4 n+j-2}=v_{j-2} \prod_{m=0}^{n-1} \frac{u_{\tau(2+j)-2} v_{\tau(2+j)}}{u_{\tau(j)} v_{\tau(j)-2}} \frac{A^{2 m+\left\lfloor\frac{j}{2}\right\rfloor}+B u_{\tau(j)} v_{\tau(j)+(-2)} \sum_{l=0}^{2 m+\left\lfloor\frac{j}{2}\right\rfloor-1} A^{l}}{C^{2 m+\left\lfloor\frac{2+j}{2}\right\rfloor}+D u_{\tau(2+j)+(-2)} v_{\tau(2+j)} \sum_{l=0}^{\left\lfloor\frac{2+j}{2}\right\rfloor-1} C^{l}},
$$

where $j=0,1,2,3$. For the sake of clarity, we can rewrite (47) and (48) in expanded forms as follows:

$$
\begin{gathered}
u_{4 n-2}=u_{-2} \prod_{m=0}^{n-1} \frac{u_{0} v_{-2}}{u_{-2} v_{0}} \frac{C^{2 m}+D u_{-2} v_{0} \sum_{l=0}^{2 m-1} C^{l}}{A^{2 m+1}+B u_{0} v_{-2} \sum_{l=0}^{2 m} A^{l}}, \\
u_{4 n-1}=u_{-1} \prod_{m=0}^{n-1} \frac{u_{1} v_{-1}}{u_{-1} v_{1}} \frac{C^{2 m}+D u_{-1} v_{1} \sum_{l=0}^{2 m-1} C^{l}}{A^{2 m+1}+B u_{1} v_{-1} \sum_{l=0}^{2 m} A^{l}}, \\
u_{4 n}=u_{0} \prod_{m=0}^{n-1} \frac{u_{0} v_{-2}}{u_{-2} v_{0}} \frac{C^{2 m+1}+D u_{-2} v_{0} \sum_{l=0}^{2 m} C^{l}}{A^{2 m+2}+B u_{0} v_{-2} \sum_{l=0}^{2 m+1} A^{l}}, \\
u_{4 n+1}=u_{1} \prod_{m=0}^{n-1} \frac{u_{1} v_{-1}}{u_{-1} v_{1}} \frac{C^{2 m+1}+D u_{-1} v_{1} \sum_{l=0}^{2 m} C^{l}}{A^{2 m+2}+B u_{1} v_{-1} \sum_{l=0}^{2 m+1} A^{l}},
\end{gathered}
$$




$$
\begin{gathered}
v_{4 n-2}=v_{-2} \prod_{m=0}^{n-1} \frac{u_{-2} v_{0}}{u_{0} v_{-2}} \frac{A^{2 m}+B u_{0} v_{-2} \sum_{l=0}^{2 m-1} A^{l}}{C^{2 m+1}+D u_{-2} v_{0} \sum_{l=0}^{2 m} C^{l}}, \\
v_{4 n-1}=v_{-1} \prod_{m=0}^{n-1} \frac{u_{-1} v_{1}}{u_{1} v_{-1}} \frac{A^{2 m}+B u_{1} v_{-1} \sum_{l=0}^{2 m-1} A^{l}}{C^{2 m+1}+D u_{-1} v_{1} \sum_{l=0}^{2 m} C^{l}}, \\
v_{4 n}=v_{0} \prod_{m=0}^{n-1} \frac{u_{-2} v_{0}}{u_{0} v_{-2}} \frac{A^{2 m+1}+B u_{0} v_{-2} \sum_{l=0}^{2 m} A^{l}}{C^{2 m+2}+D u_{-2} v_{0} \sum_{l=0}^{2 m+1} C^{l}}, \\
v_{4 n+1}=v_{1} \prod_{m=0}^{n-1} \frac{u_{-1} v_{1}}{u_{1} v_{-1}} \frac{A^{2 m+1}+B u_{1} v_{-1} \sum_{l=0}^{2 m} A^{l}}{C^{2 m+2}+D u_{-1} v_{1} \sum_{l=0}^{2 m+1} C^{l}} .
\end{gathered}
$$

Theorem 1. The following system of equations

$$
u_{n+1}=\frac{u_{n-1} v_{n-3}}{v_{n-1}\left(A+B u_{n-1} v_{n-3}\right)} \text { and } v_{n+1}=\frac{u_{n-3} v_{n-1}}{u_{n-1}\left(C+D u_{n-3} v_{n-1}\right)}
$$

has a 2-periodic solution if $u_{-3}=u_{-1}, v_{-3}=v_{-1}, u_{-3} v_{-3}=u_{-2} v_{-2}=\frac{(1-A)}{B}$ and $A=C$, $B=D \neq 0$.

Proof. Let $u_{-3}=u_{-1}, v_{-3}=v_{-1}$ and $u_{-3} v_{-3}=u_{-2} v_{-2}=\frac{(1-A)}{B}$, where $A=C, B=D$ in the exact Equation (49). Then,

$$
\begin{aligned}
u_{4 n-2} & =u_{-2} \prod_{m=0}^{n-1} \frac{C^{2 m}+(1-C) \sum_{l=0}^{2 m-1} C^{l}}{A^{2 m+1}+(1-A) \sum_{l=0}^{2 m} A^{l}} \\
& =u_{-2} .
\end{aligned}
$$

Following the same procedure as above on Equations (50)-(56) gives $u_{4 n-1}=u_{-1}$; $u_{4 n}=u_{0} ; u_{4 n+1}=u_{1}$ and $v_{4 n-1}=v_{-1} ; v_{4 n}=v_{0} ; v_{4 n+1}=v_{1}$.

Below is a graph that illustrates the theorem above. Figure 1 is when we let $A=C=2, B=D=-0.125, u_{1}=4, u_{0}=1, v_{1}=2$ and $v_{0}=8$ into the system of Equation (57). 


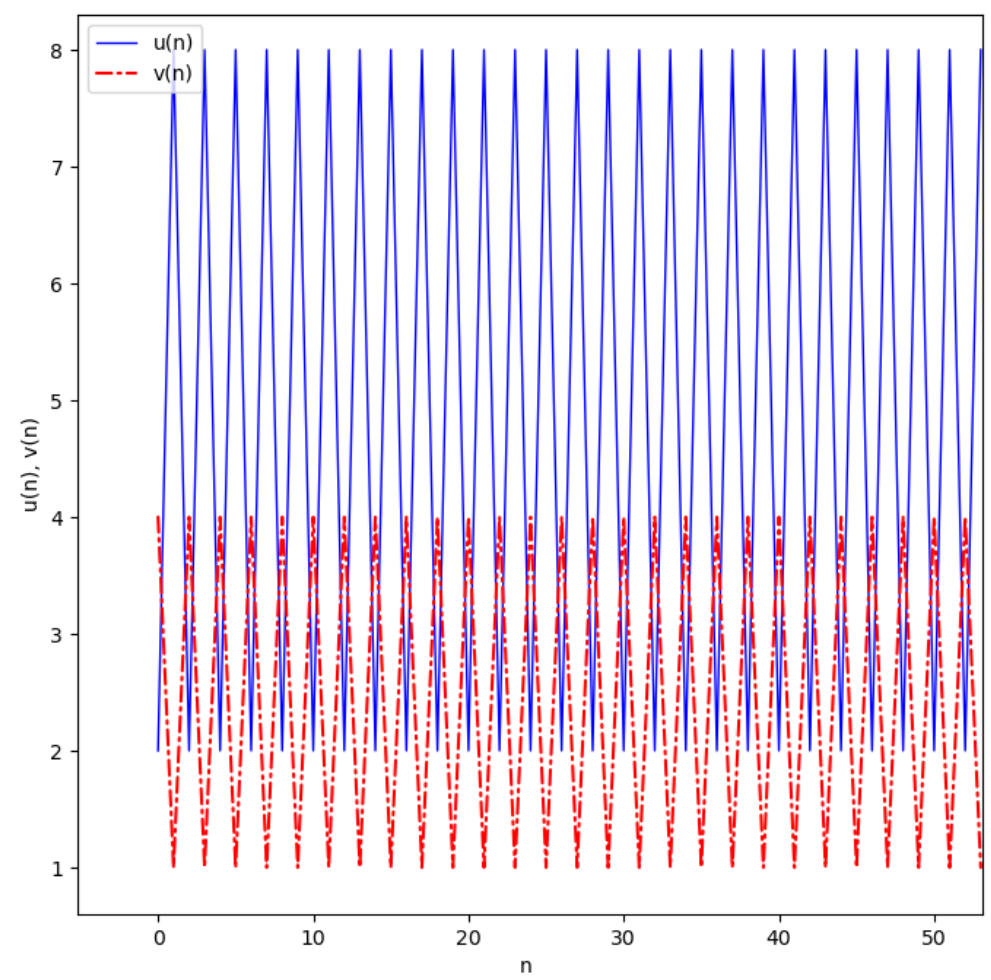

Figure 1. Plof of $u_{n+1}=\frac{u_{n-1} v_{n-3}}{v_{n-1}\left(2-0.125 u_{n-1} v_{n-3}\right)}, \quad v_{n+1}=\frac{u_{n-3} v_{n-1}}{u_{n-1}\left(2-0.125 u_{n-3} v_{n-1}\right)}$.

If we let $A=B=C=1$ and $D=-1$, then Equations (49)-(56) reduce to Theorem 4 in [11] as shown below:

$$
\begin{aligned}
u_{4 n-2}= & u_{-2} \prod_{m=0}^{n-1} \frac{u_{0} v_{-2}}{u_{-2} v_{0}} \frac{(1)^{2 m}-u_{-2} v_{0} \sum_{i=1}^{2 m}(1)^{i}}{(1)^{2 m+1}+u_{0} v_{-2} \sum_{i=1}^{2 m+1}(1)^{i}} \\
= & u_{-2} \prod_{m=0}^{n-1} \frac{u_{0} v_{-2}}{u_{-2} v_{0}} \frac{\left(-\left((2 m) u_{-2} v_{0}-1\right)\right)}{1+(2 m+1) u_{0} v_{-2}} \\
= & \frac{(-1)^{n}\left(u_{0}\right)^{n}\left(v_{-2}\right)^{n} \prod_{m=0}^{n-1}\left[(2 m) u_{-2} v_{0}-1\right]}{\left(u_{-2}\right)^{n-1}\left(v_{0}\right)^{n} \prod_{m=0}^{n-1}\left[(2 m+1) u_{0} v_{-2}+1\right]} .
\end{aligned}
$$

Just as above, we similarly obtain the following:

$$
\begin{gathered}
u_{4 n-1}=\frac{(-1)^{n}\left(u_{-1}\right)^{n+1}\left(v_{-3}\right)^{n} \prod_{m=0}^{n-1}\left[(2 m+1) u_{-3} v_{-1}-1\right]}{\left(u_{-3}\right)^{n}\left(v_{-1}\right)^{n} \prod_{m=0}^{n-1}\left[(2 m+2) u_{-1} v_{-3}+1\right]}, \\
u_{4 n}=\frac{(-1)^{n}\left(u_{0}\right)^{n+1}\left(v_{-2}\right)^{n} \prod_{m=0}^{n-1}\left[(2 m+1) u_{-2} v_{0}-1\right]}{\left(u_{-2}\right)^{n}\left(v_{0}\right)^{n} \prod_{m=0}^{n-1}\left[(2 m+2) u_{0} v_{-2}+1\right]}
\end{gathered}
$$




$$
\begin{gathered}
u_{4 n+1}=\frac{(-1)^{n}\left(u_{1}\right)^{n+1}\left(v_{-1}\right)^{n} \prod_{m=0}^{n-1}\left[(2 m+1) u_{-1} v_{1}-1\right]}{\left(u_{-1}\right)^{n}\left(v_{1}\right)^{n} \prod_{m=0}^{n-1}\left[(2 m+2) u_{1} v_{-1}+1\right]}, \\
v_{4 n-2}=\frac{(-1)^{n}\left(u_{-2}\right)^{n}\left(v_{0}\right)^{n} \prod_{m=0}^{n-1}\left[(2 m) u_{0} v_{-2}+1\right]}{\left(u_{0}\right)^{n}\left(v_{-2}\right)^{n} \prod_{m=0}^{n-1}\left[(2 m+1) u_{-2} v_{0}-1\right]}, \\
v_{4 n-1}=\frac{(-1)^{n}\left(u_{-3}\right)^{n}\left(v_{-1}\right)^{n+1} \prod_{m=0}^{n-1}\left[(2 m+1) u_{-1} v_{-3}+1\right]}{\left(u_{-1}\right)^{n}\left(v_{-3}\right)^{n} \prod_{m=0}^{n-1}\left[(2 m+2) u_{-3} v_{-1}-1\right]}, \\
v_{4 n}=\frac{(-1)^{n}\left(u_{-2}\right)^{n}\left(v_{0}\right)^{n+1} \prod_{m=0}^{n-1}\left[(2 m+1) u_{0} v_{-2}+1\right]}{\left(u_{0}\right)^{n}\left(v_{-2}\right)^{n} \prod_{m=0}^{n-1}\left[(2 m+2) u_{-2} v_{0}-1\right]} \\
v_{4 n+1}=\frac{(-1)^{n}\left(u_{-1}\right)^{n}\left(v_{1}\right)^{n+1} \prod_{m=0}^{n-1}\left[(2 m+1) u_{1} v_{-1}+1\right]}{\left(u_{1}\right)^{n}\left(v_{-1}\right)^{n} \prod_{m=0}^{n-1}\left[(2 m+2) u_{-1} v_{1}-1\right]} .
\end{gathered}
$$

Similarly, if we let $A=B=C=D=1$, then Equations (49)-(56) reduce to Theorem 1 in [11], as shown below:

$$
u_{4 n-2}=u_{-2} \prod_{m=0}^{n-1} \frac{u_{0} v_{-2}}{u_{-2} v_{0}} \frac{(1)^{2 m}+(1) u_{-2} v_{0} \sum_{i=1}^{2 m}(1)^{i+1}}{(1)^{2 m+1}+(1) u_{0} v_{-2} \sum_{i=1}^{2 m+1}(1)^{i+1}}
$$

In the above equation, we performed index shifting. Now,

$$
\begin{aligned}
u_{4 n-2} & =u_{-2} \prod_{m=0}^{n-1} \frac{u_{0} v_{-2}}{u_{-2} v_{0}} \frac{1+(2 m) u_{-2} v_{0}}{1+(2 m+1) u_{0} v_{-2}} \\
& =u_{-2} \prod_{m=0}^{n-1} \frac{u_{0} v_{-2}}{u_{-2} v_{0}} \frac{1+(2 m) u_{-2} v_{0}}{1+(2 m+1) u_{0} v_{-2}} .
\end{aligned}
$$

Thus, (49) simplifies to

$$
u_{4 n-2}=\frac{\left(u_{0}\right)^{n}\left(v_{-2}\right)^{n} \prod_{m=0}^{n-1}\left[(2 m) u_{-2} v_{0}+1\right]}{\left(u_{-2}\right)^{n-1}\left(v_{0}\right)^{n} \prod_{m=0}^{n-1}\left[(2 m+1) u_{0} v_{-2}+1\right]} .
$$

Following the same procedure as above, Equations (50)-(56), respectively, result in the following equations:

$$
u_{4 n-1}=\frac{\left(u_{-1}\right)^{n+1}\left(v_{-3}\right)^{n} \prod_{m=0}^{n-1}\left[(2 m+1) u_{-3} v_{-1}+1\right]}{\left(u_{-3}\right)^{n}\left(v_{-1}\right)^{n} \prod_{m=0}^{n-1}\left[(2 m+2) u_{-1} v_{-3}+1\right]}
$$




$$
\begin{gathered}
u_{4 n}=\frac{\left(u_{0}\right)^{n+1}\left(v_{-2}\right)^{n} \prod_{m=0}^{n-1}\left[(2 m+1) u_{-2} v_{0}+1\right]}{\left(u_{-2}\right)^{n}\left(v_{0}\right)^{n} \prod_{m=0}^{n-1}\left[(2 m+2) u_{0} v_{-2}+1\right]}, \\
u_{4 n+1}=\frac{\left(u_{-1}\right)^{n+1}\left(v_{-3}\right)^{n} \prod_{m=0}^{n-1}\left[(2 m+1) u_{-3} v_{-1}+1\right]}{\left(u_{-3}\right)^{n}\left(v_{-1}\right)^{n} \prod_{m=0}^{n-1}\left[(2 m+2) u_{-1} v_{-3}+1\right]} \\
v_{4 n-2}=\frac{\left(u_{-2}\right)^{n}\left(v_{0}\right)^{n} \prod_{m=0}^{n-1}\left[(2 m) u_{0} v_{-2}+1\right]}{\left(u_{0}\right)\left(v_{-2}\right)^{n-1} \prod_{m=0}^{n-1}\left[(2 m+1) u_{-2} v_{0}+1\right]} \\
v_{4 n+1}=\frac{\left(u_{-3}\right)^{n}\left(v_{-1}\right)^{n+1} \prod_{m=0}^{n-1}\left[(2 m+1) u_{-1} v_{-3}+1\right]}{\left(u_{-1}\right)^{n}\left(v_{-3}\right)^{n} \prod_{m=0}^{n-1}\left[(2 m+2) u_{-3} v_{-1}+1\right]} \\
v_{4 n}=\frac{\left(u_{-2}\right)^{n}\left(v_{0}\right)^{n+1} \prod_{m=0}^{n-1}\left[(2 m+1) u_{0} v_{-2}+1\right]}{\left(u_{0}\right)^{n}\left(v_{-2}\right)^{n} \prod_{m=0}^{n-1}\left[(2 m+2) u_{-2} v_{0}+1\right]},
\end{gathered}
$$

When $k=3$ in (5), the resulting $O \Delta E$ s are

$$
u_{n+1}=\frac{u_{n-1} v_{n-4}}{v_{n-2}\left(a+b u_{n-1} v_{n-4}\right)} \quad \text { and } \quad v_{n+1}=\frac{u_{n-4} v_{n-1}}{u_{n-2}\left(c+d u_{n-4} v_{n-1}\right)}
$$

Example 1. We get the illustration below (see Figure 2) when we let $a=c=-1$, $b=d=1, u_{-4}=7, u_{-3}=5, u_{-2}=-0.0971, u_{-1}=7, u_{0}=1, v_{-4}=3$, $v_{-3}=9, v_{-2}=0.01, v_{-1}=-3$ and $v_{0}=2$ in (76).

Example 2. Letting $a=c=1, b=d=-1, u_{-4}=0.88, u_{-3}=0.35, u_{-2}=0.79$, $u_{-1}=0.95, u_{0}=0.31, v_{-4}=0.77, v_{-3}=0.33, v_{-2}=0.16, v_{-1}=0.35$ and $v_{0}=0.75$ in (76) yields Figure 3. 


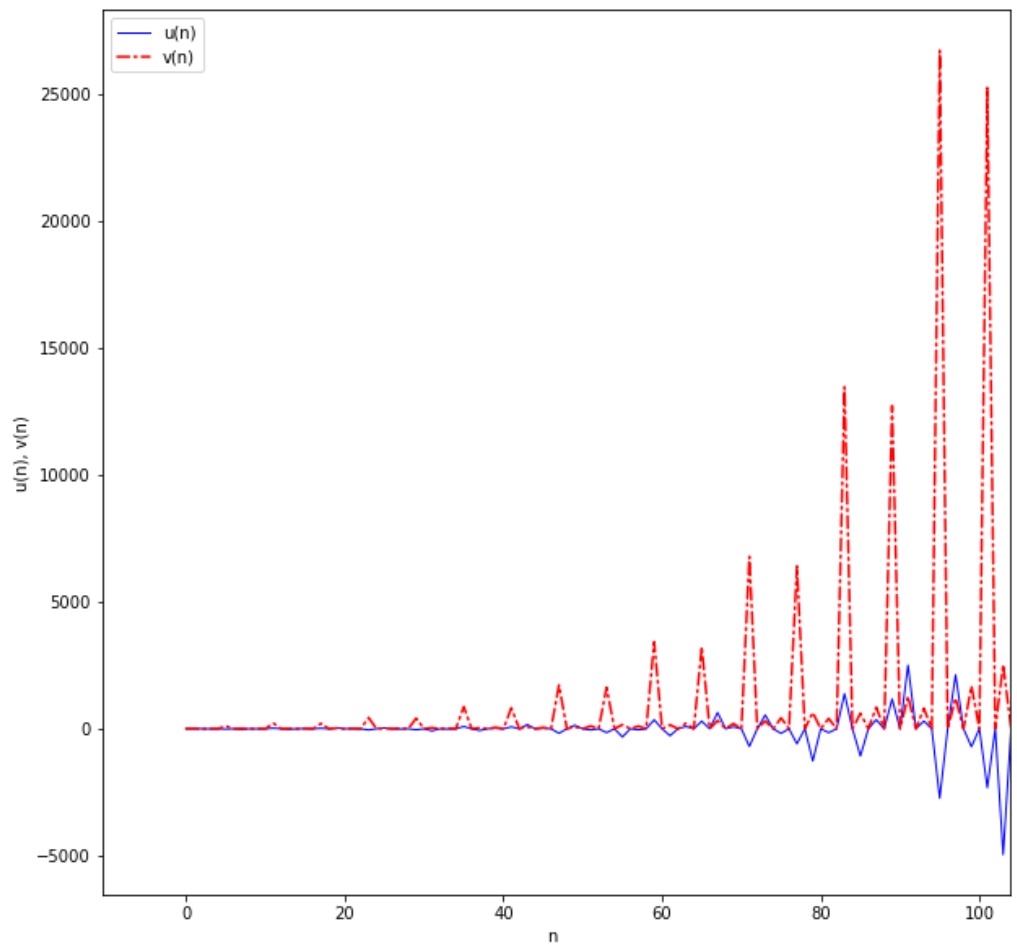

Figure 2. Plot of $u_{n+1}=\frac{u_{n-1} v_{n-4}}{v_{n-2}\left(-1+u_{n-1} v_{n-4}\right)}, v_{n+1}=\frac{u_{n-4} v_{n-1}}{u_{n-2}\left(-1+u_{n-4} v_{n-1}\right)}$.

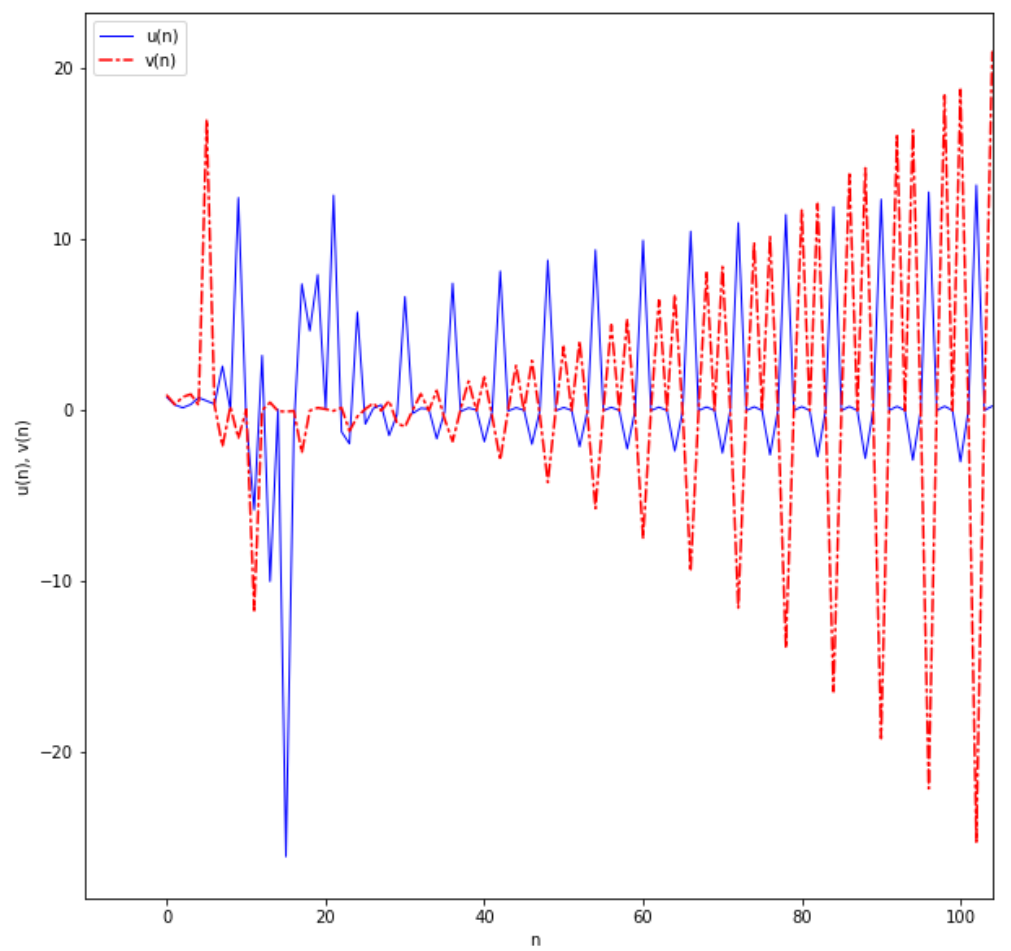

Figure 3. Plot of $u_{n+1}=\frac{u_{n-1} v_{n-4}}{v_{n-2}\left(1-u_{n-1} v_{n-4}\right)}, v_{n+1}=\frac{u_{n-4} v_{n-1}}{u_{n-2}\left(1-u_{n-4} v_{n-1}\right)}$.

\section{Conclusions}

In this study, we looked at a system of $(k+2)$ th order ordinary difference equations. We investigated these equations by finding the symmetry generators (29). We then used the canonical coordinates to find the invariants in (31) of which led us to get the closed form solutions (33) and (34). Performing iterations resulted in (36), where we used the floor func- 
tion definition and back-shifted the resulting equations to obtain the solutions (43) and (44) of (5). We performed a detailed study of the case where $k=2$. The reason for studying this case is that we wanted to show that the work done in the articles [11,12] are special cases of our results. In fact, for different combinations of values of $A, B, C$ and $D$, which we get from the articles [11,12], we obtain the following important results:

Equations (49)-(56) are reduced to equations in Theorem 2 in [11] when we set $A=B=1$ and $C=D=-1$.

Equations (49)-(56) are reduced to equations in Theorem 3 in [11] when we set $A=B=D=1$ and $C=-1$.

Equations (49)-(56) are reduced to equations in Theorem 4 in [11] when we set $A=B=C=1$ and $D=-1$.

Equations (49)-(56) are reduced to equations in Theorem 1 in [12] when we set $A=C=1$ and $B=D=-1$.

Equations (49)-(56) are reduced to equations in Theorem 2 in [12] when we set $A=C=-1$ and $B=D=1$.

Equations (49)-(56) are reduced to equations in Theorem 3 in [12] when we set $A=D=1$ and $B=C=-1$.

Equations (49)-(56) are reduced to Theorem 4 in [12] when we set $A=D=-1$ and $B=C=1$. We also stated and provided the proof for the existence of 2-periodic solutions.

Author Contributions: Both authors contribute equally to the manuscript. All authors have read and agreed to the published version of the manuscript.

Funding: This research received no external funding.

Institutional Review Board Statement: Not applicable.

Informed Consent Statement: Not applicable.

Data Availability Statement: Not applicable.

Conflicts of Interest: The authors declare no conflict of interest.

\section{References}

1. Quadling, D.; Klein, F.; Lie, S. Evolution of the idea of symmetry in the nineteenth century, by IM Yaglom. Translated by Sergei Sossinsky and edited by Hardy Grant and Abe Shenitzer. Math. Gaz. 1988, 72, 341-342. [CrossRef]

2. Lie, S. Vorlesungen über Differentialgleichungen: Mit Bekannten Infinitesimalen Transformationen; BG Teubner: Leipzig, Germany, 1891.

3. Sedov, L.I.; Volkovets, A.G. Similarity and Dimensional Methods in Mechanics, 10th ed.; CRC Press: Boca Raton, FL, USA, 1961.

4. Birkhoff, G. Hydrodynamics, a study in logic, fact, and similitude. Bull. Am. Math. Soc. 1951, 57, 497-499.

5. Maeda, S. The similarity method for difference equations. IMA J. Appl. Math. 1987, 38, 129-134. [CrossRef]

6. Maeda, S. Canonical structure and symmetries for discrete systems. Math. Jpn. 1980, 25, 405-420.

7. Levi, D.; Winternitz, P. Continuous symmetries of discrete equations. Phys. Lett. A 1991, 152, 335-338. [CrossRef]

8. Hydon, P.E. Difference Equations by Differential Equation Methods; Cambridge University Press: Cambridge, UK, 2014.

9. Quispel, G.; Sahadeva, R. Lie symmetries and the integration of difference equations. Phys. Lett. A 1993, 184, 64-70. [CrossRef]

10. Banasiak, J. Mathematical Modelling in One Dimension: An Introduction via Difference and Differential Equation; Cambridge University Press: Cambridge, UK, 2013.

11. Almatrafi, M.B.; Elsayed, E.M. Solutions and formulae for some systems of difference equations. MathLAB J. 2018, 1, 356-369.

12. Almatrafi, M.B. Analysis of Solutions of Some Discrete Systems of Rational Difference Equations. J. Comput. Anal. Appl. 2020 29, 355-368.

13. Folly-Gbetoula, M.; Nyirenda, D. A generalised two-dimensional system of higher order recursive sequences. J. Differ. Equ. Appl. 2020, 26, 244-260. [CrossRef]

14. Hydon, P.E. Symmetry Methods for Differential Equations: A Beginner's Guide; Cambridge University Press: Cambridge, UK, 2000; Volume 22.

15. Joshi, N.; Vassiliou, P. The existence of Lie Symmetries for First-Order Analytic Discrete Dynamical Systems. J. Math. Anal. Appl. 1995, 195, 872-887. [CrossRef] 